\title{
Concentração e quantidade de nutrientes em mudas de Teca produzidas em substratos $\operatorname{orgânicos}^{1}$
}

\author{
Paulo André Trazzi ${ }^{2}$; William Macedo Delarmelina ${ }^{3}$; Marcos Vinicius Winckler Caldeira ${ }^{4}$
}

\begin{abstract}
Resumo: Os resíduos industriais, urbanos ou agropecuários podem ser utilizados como fonte de nutrientes visando contribuir para a redução dos custos de produção de mudas. O objetivo deste trabalho foi determinar a concentração e a quantidade de nutrientes em mudas de Tectona grandis produzidas em substrato orgânico. As mudas foram produzidas em tubetes com capacidade volumétrica de $280 \mathrm{~cm}^{3}$, preenchidos com dois grupos de resíduos e constituindo dois estudos. O primeiro constituiu em formular substratos com biossólido (BIO) associado à casca de arroz carbonizada (CAC) ou à fibra de coco (FB) triturada nas proporções 80:20, 60:40, 40:60, 20:80 (v:v), e também com 100\% de BIO, formando nove tratamentos a base de $\mathrm{BIO}$ submetidos à comparação do tratamento com o substrato comercial florestal. $\mathrm{O}$ segundo estudo caracterizou-se por utilizar proporções (v:v:v) de 15, 25 ou 35\% de esterco bovino (EB), cama de frango (CF) ou esterco de codorna (EC) associados à terra de subsolo $(60,50 \mathrm{ou} 40 \%$, respectivamente) e a $25 \%$ de substrato comercial florestal, formando um conjunto de nove tratamentos. Considerando as concentrações e quantidades de nutrientes, os maiores valores foram obtidos no tratamento com $80 \% \mathrm{BIO}+20 \%$ de CAC, para os tratamentos com biossólido. Para o estudo com estercos de animais, a maior quantidade de nutrientes foi observada no tratamento com 35\% de CF. O BIO com 60 e $80 \%$ associado à CAC ou a FC promoveu os maiores ganhos em altura, diâmetro, massa seca da parte aérea e radicular, no experimento com biossólido.
\end{abstract}

Palavras - chave: Tectona grandis; Lodo de esgoto; Produção de mudas; Nutrição florestal.

\section{Concentration and amount of nutrients in Teca seedlings produced in organic substrates}

\begin{abstract}
Industrial, urban and agricultural residues can be used as a source of nutrients to contribute to reducing the costs of seedling production. The aim of this study was to determine the concentraton and the amount of nutrients in seedlings of Tectona grandis produced in organic substrate. The seedlings were grown in tubes with volumetric capacity of $280 \mathrm{~cm}^{3}$, filled with two groups of residues and constituting two studies. The first consisted in formulating substrates with biosolids associated with carbonized rice hull or shredded coconut fiber in the proportions 80:20, 60:40, 40:60, 20:80 (v:v), and with 100\% BIO forming the basis of nine treatments BIO undergoing treatment compared with the commercial forestry substrate. The second study was characterized by using ratios (v:v:v) for 15,25 or $35 \%$ of cattle manure, poultry litter or quail manure associated with subsoil $(60,50$ or $40 \%$, respectively) and $25 \%$ commercial forestry substrate forming a set of nine treatments were compared to treatment with commercial forestry substrate. The substrates formed these treatments were subjected to chemical and physical analyzes. To evaluate the nutritionally seedlings, shoots was subjected to chemical analysis. Considering the concentrations and amounts of nutrients, the highest were obtained in the treatment with $80 \% \mathrm{BIO}+20 \% \mathrm{CAC}$, for the treatments with biosolid. For the study of animal manure, the largest amount of nutrients was observed in the treatment with $35 \%$ CF. The BIO 60 and $80 \%$ associated with CAC or FC promoted greater gains in height, diameter, dry mass of shoots and roots, in the experiment with sewage sludge.
\end{abstract}

Keywords: Tectona grandis; Sewage sludge; Seedlings production, Forest nutrition.

\footnotetext{
${ }^{1}$ Recebido em 27.06.2014 e aceito para publicação como artigo científico em: 29.09.2014.

${ }^{2}$ Engenheiro Florestal, Doutorando em Engenharia Florestal, Universidade Federal do Paraná, Rua Pref. Lhotário Meissner, 900, Jardim Botânico, CEP: 80210- 170 - Curitiba/PR. e-mail: <patrazzi@ hotmail.com>.

${ }^{3}$ Engenheiro Florestal, Mestrando em Ciências Florestais, Departamento de Ciências Florestais e da Madeira, Centro de Ciências Agrárias, Universidade Federal do Espírito Santo. Alto Universitário, s/n - Guararema. CP 16, CEP: 29500-000- Alegre/ES. e-mail: <williamdm@hotmail.com>.

${ }^{4}$ Engenheiro Florestal, D.Sc., Professor do Departamento de Ciências Florestais e da Madeira, Centro de Ciências Agrárias, Universidade Federal do Espírito Santo. Alto Universitário, s/n - Guararema. CP 16, CEP: 29500-000 - Alegre/ES. Bolsista de produtividade em pesquisa do CNPq. e-mail: <mvwcaldeira@gmail.com>; <caldeiaramv@pq.cnpq.br>.
} 


\section{Introdução}

O Brasil possui uma crescente demanda por produtos de origem florestal, implicando o aumento do consumo de fertilizantes na produção de mudas e na ocasião de plantio. Com base nisso, a utilização de componentes orgânicos, tal como o biossólido, casca de arroz carbonizada, fibra de coco, esterco bovino, cama de frango e esterco de codorna podem contribuir para redução do uso de fertilizantes minerais. Além disso, quando utilizado como substrato para a produção de mudas, se torna uma alternativa para a redução de custos, tendo em vista sua disponibilidade e facilidade de aquisição.

Vários fatores devem ser considerados para se obter florestas com alto padrão de qualidade, sendo um dos principais a qualidade das mudas utilizadas no plantio, que devem apresentar adequada nutrição, serem vigorosas, resistentes ao estresse do transplante e livre de pragas e doenças, assegurando boa adaptação e crescimento após o plantio (CRUZ et al., 2004). A obtenção de mudas de boa qualidade exige a utilização de substrato que forneça os nutrientes necessários ao pleno desenvolvimento da planta, pois o substrato exerce uma influência marcante na arquitetura do sistema radicular e no estado nutricional das plantas, afetando a qualidade das mudas.

Cunha et al. (2006), analisando o efeito de diferentes substratos sobre o crescimento de mudas de Acacia sp. em diferentes substratos, concluíram que as mudas desenvolvidas no substrato com apenas biossólido em sua composição, foram as que acumularam mais $\mathrm{Ne}$ $\mathrm{Ca}$, principalmente quando inoculadas e que houve tendência de maior acúmulo de $\mathrm{P}, \mathrm{K}$ e $\mathrm{Mg}$ em mudas crescidas no substrato com esterco bovino. Resultados promissores também foram relatados por Rocha et al. (2013) com a utilização do composto de biossólido sem ou com material estruturante até um percentual de $60 \%$, como substrato para produção de mudas clonais de Eucalyptus sp.

Tectona grandis Linn. F. (teca) é originária do continente asiático, estando sua área de ocorrência confinada entre Floresta Úmida e Decídua Árida Mista. Essa espécie foi introduzida na região Norte do Brasil em 1994, com a finalidade de cumprir a reposição florestal obrigatória em atendimento à legislação ambiental vigente (FIGUEIREDO, 2005). No Brasil, a área plantada vem crescendo nos últimos anos, sendo atualmente superior a $67 \mathrm{mil}$ ha, localizada principalmente no Mato Grosso (ABRAF, 2013).

O trabalho foi realizado com o objetivo de determinar a concentração e a quantidade de nutrientes em mudas de Tectona grandis produzidas em substratos orgânicos.

\section{Material e métodos}

Localização

Os experimentos foram desenvolvidos no Viveiro Florestal do Departamento de Ciências Florestais e da Madeira do Centro de Ciências Agrárias da Universidade Federal do Espírito Santo (DCFM/CCA-UFES), Alegre/ES, nas coordenadas geográficas de $20^{\circ} 45^{\prime} \mathrm{S}$ e $41^{\circ} 31^{\prime}$ $\mathrm{W}$, com altitude média de $277 \mathrm{~m}$. O clima enquadra-se no tipo Cwa (inverno seco e verão chuvoso), de acordo com a classificação de Köppen, com precipitação anual média de 1104 mm e temperatura média anual de $24,1^{\circ} \mathrm{C}$, com máximas diárias de $31^{\circ} \mathrm{C}$ e mínimas de $20,2^{\circ} \mathrm{C}$ (ESPÍRITO SANTO, 1994).

\section{Delineamento Experimental}

As mudas foram produzidas em dois grupos de substratos, onde os tratamentos testados foram constituídos com biossólido ou com estercos de animais, constituindo dois experimentos instalados concomitantemente.

Para cada experimento, foi utilizado um delineamento inteiramente casualizado constituindo dez tratamentos, com seis repetições por tratamento sendo cada repetição constituída por quatro plantas, totalizando vinte e quatro mudas por tratamento.

As variáveis analisadas foram submetidas à análise estatística através do teste $\mathrm{F}$, e quando 
significativo, as médias foram comparadas pelo teste Scott-Knott a nível de 5\% de probabilidade de erro, por meio do software SPSS19®.

No primeiro experimento foram utilizados diferentes proporções (\%) de biossólido (BIO) com casca de arroz carbonizada (CAC) ou fibra de coco triturada (FC). Utilizou-se o substrato comercial florestal (SC) como tratamento testemunha. Os tratamentos foram compostos pelas seguintes proporções de biossólido, casca de arroz carbonizada e fibra de coco (BIO:CAC:FC): B1 (100:00:00); B2 (80:20:00); B3 (60:40:00); B4 (40:60:00); B5 (20:80:00); B6 (80:00:20); B7 (60:00:40); B8 (40:00:60); B9 (20:00:80); S10 (100\% substrato comercial florestal).

\section{Caracterização dos Substratos}

O biossólido utilizado no experimento foi doado pela Foz do Brasil S.A. (ETE de Cachoeiro de Itapemirim-ES). Na Tabela 1, observa-se que as quantidades de metais pesados poluentes presentes no biossólido são baixas, e de acordo com a resolução CONAMA 375/2006 (BRASIL, 2006), esse material está apto para uso em ambientes agrícolas.

No segundo experimento, foram utilizados estercos de animais: esterco de codorna (EC), esterco bovino (EB) e cama de frango (CF). Estes foram associados a diferentes proporções de terra de subsolo (TS). Para dar mais consistência e porosidade ao substrato, foi utilizada uma fração de $25 \%$ de substrato comercial florestal para todas as combinações entre estercos de animais e terra de subsolo. Os tratamentos foram compostos pelas seguintes proporções de substrato comercial florestal, terra de subsolo, esterco bovino, cama de frango e esterco de codorna (SC:TS:EB:CF:EC): M1 (25:40:35:00:00); M2 (25:50:25:00:00); M3 (25:60:15:00:00); M4 (25:40:00:35:00); M5 (25:50:00:25:00); M6 (25:60:00:15:00); M7 (25:40:00:00:35); M8 (25:50:00:00:25); M9 (25:60:00:00:15); S10 (100\% substrato comercial florestal).

Tabela 1. Análise química do biossólido de filtro anaeróbico oriundo da estação de tratamento de esgoto de Cachoeiro de Itapemirim, ES, 2011.

Table 1. Chemical analysis os sewage sludge anaerobic filter derived from the sewage treatment station of Cachoeiro de Itapemirim, ES, 2011.

\begin{tabular}{lll}
\hline Parâmetros & Resultados Analíticos & CONAMA 375/2006 \\
\hline Arsênio & $*<0,5 \mathrm{mg} \mathrm{dm}^{-3}$ & $41 \mathrm{mg} \mathrm{Kg}^{-1}$ \\
Bário & $156 \mathrm{mg} \mathrm{dm}^{-3}$ & $1300 \mathrm{mg} \mathrm{Kg}^{-1}$ \\
Cádmio & $<0,053 \mathrm{mg} \mathrm{dm}^{-3}$ & $39 \mathrm{mg} \mathrm{Kg}^{-1}$ \\
Chumbo & $29 \mathrm{mg} \mathrm{dm}^{-3}$ & $300 \mathrm{mg} \mathrm{Kg}^{-1}$ \\
Cobre & $98 \mathrm{mg} \mathrm{dm}$ & -3 \\
Cromo & $26 \mathrm{mg} \mathrm{dm}$ & \\
Molibdênio & $3,5 \mathrm{mg} \mathrm{dm}^{-3}$ & $1500 \mathrm{mg} \mathrm{Kg}^{-1}$ \\
Níquel & $11 \mathrm{mg} \mathrm{dm}^{-3}$ & $1000 \mathrm{mg} \mathrm{Kg}^{-1}$ \\
Selênio & $<0,5 \mathrm{mg} \mathrm{dm}^{-3}$ & $50 \mathrm{mg} \mathrm{Kg}^{-1}$ \\
Zinco & $409 \mathrm{mg} \mathrm{dm}^{-3}$ & $420 \mathrm{mg} \mathrm{Kg}^{-1}$ \\
Fósforo Total & $4128 \mathrm{mg} \mathrm{dm}^{-3}$ & $100 \mathrm{mg} \mathrm{Kg}^{-1}$ \\
pH (Suspensão a 5\%) & $5,2 \mathrm{mg} \mathrm{dm}^{-3}$ & $2800 \mathrm{mg} \mathrm{Kg}^{-1}$ \\
Enxofre & $1,3 \%$ & - \\
Nitrogênio Total (Kjeldahl) & $5646 \mathrm{mg} \mathrm{dm}^{-3}$ & - \\
Nitrogênio Amoniacal & $60 \mathrm{mg} \mathrm{dm}^{-3}$ & - \\
Carbono Orgânico Total & $16 \%$ & - \\
Potássio & $1623 \mathrm{mg} \mathrm{dm}^{-3}$ & - \\
Sódio & $399 \mathrm{mg} \mathrm{dm}^{-3}$ & - \\
\hline
\end{tabular}

*Valores em base seca e resultados fornecidos pela Foz do Brasil S.A.; ${ }^{1}$ Limites máximos de concentração exigidos pelo CONAMA 375/2006. 
Antes de ser utilizado na formulação dos substratos, o biossólido foi peneirado sobre malha de $3 \mathrm{~mm}$ depois de permanecer por cerca de 30 dias em ambiente aberto.

A casca de arroz carbonizada e a fibra de coco triturada foram doadas pela Fibria Celulose S.A. (Unidade Aracruz-ES). Esses materiais são normalmente utilizados como componentes de substratos para produção de mudas de Eucalytptus.

O esterco bovino e o esterco de codorna foram coletados na Área Experimental I do CCA/UFES. A cama de frango foi doada pelo Instituto Federal de Educação, Ciência e Tecnologia do Espírito Santo, campus de Alegre, ES. O esterco bovino, a cama de frango e o esterco de codorna passaram por processo de estabilização biológica no viveiro, durante cerca de 30, 60 e 90 dias, respectivamente, onde permaneceram em local aberto. Depois de curtidos passaram por peneiras de $3 \mathrm{~mm}$ de malha.

A terra de subsolo utilizada como componente de substrato foi um Latossolo Vermelho Amarelo (EMBRAPA, 2006) coletado na profundidade de $20-40 \mathrm{~cm}$, na Área Experimental I CCA/UFES. Para compor o substrato, a terra de subsolo foi peneirada em malha de $3 \mathrm{~mm}$.

Produção de mudas

As mudas foram produzidas a partir de sementes, cujos frutos foram obtidos por meio de doação da empresa Floresteca S.A. Para quebra de dormência, estes frutos foram colocados imersos em água por três noites e postos em pleno sol durantes três dias, segundo recomendações de Figueiredo et al. (2005). Em seguida, os frutos foram colocados em canteiro de areia lavada para germinação.

Seguindo os tratamentos estabelecidos, foram realizadas as misturas utilizando-se proveta graduada para medir os volumes dos componentes a serem misturados. Os substratos formados foram homogeneizados e, em seguida, amostras de cada tratamento foram separadas para posteriores análises químicas.
Os recipientes usados para a produção de mudas foram tubetes cônicos de polipropileno com dimensões de $19 \mathrm{~cm}$ de comprimento, 5,2 $\mathrm{cm}$ de diâmetro de abertura superior, $1,3 \mathrm{~cm}$ de diâmetro de abertura inferior, e capacidade volumétrica de $280 \mathrm{~cm}^{3}$.

Com os substratos de cada tratamento homogeneizados e umedecidos, os tubetes foram preenchidos realizando leve compactação manual, de forma que o substrato se acomodasse dentro dos mesmos, tornando-o consistente.

A semeadura foi feita em sementeiras com areia lavada. As plantas permaneceram por um mês nas sementeiras desde a semeadura e após atingirem altura entre cinco e sete centímetros, as plantas foram repicadas para os tubetes, preenchidos com substratos de seus, respectivos, tratamentos. Os tubetes foram acondicionados, na casa de sombra (com sombrite de 50\%), em bandejas de polipropileno.

Após a repicagem, cada bandeja foi constituída de seis repetições (24 plantas). Sessenta dias após, foi realizado o espaçamento das mudas, ficando então cada bandeja com três repetições (12 plantas). Com isso aumentou-se o espaço entre as mudas, reduzindo a competição por luz, aumentando a aeração e melhorando a eficiência da irrigação.

As mudas permaneceram na casa de sombra durante 90 dias, sendo irrigadas quatro vezes ao dia, por sistema de irrigação automático. Para dias chuvosos, a irrigação foi cessada.

Visando avaliar somente a influência dos substratos na nutrição das mudas, não houve qualquer adubação de base e de cobertura.

Análise química do tecido vegetal e dos substratos

Ao final do ciclo das mudas no viveiro (noventa dias após a repicagem), foi realizada a análise química da parte aérea (folhas + caule) das mudas para determinação da concentração dos nutrientes (Tabelas 2).

A determinação das concentrações dos nutrientes foi realizada no laboratório de Análises de Fertilizantes, Águas, Minérios, Resíduos, Solos e Plantas (LAFARSOL) do 
DCFM/CCA-UFES, Jerônimo Monteiro, ES, segundo a metodologia descrita por EMBRAPA (2009).

Tabela 2. Concentração de nutrientes dos substratos formulados com biossólido (experimento I) e esterco de animais (experimento II)

Table 2. Nutrient concentration of substrates formulated with biosolids (experiment I) and animal manure (experiment II).

Experimento I

\begin{tabular}{cccccccccccc}
\hline & $\mathrm{N}$ & $\mathrm{P}$ & $\mathrm{K}$ & $\mathrm{Ca}$ & $\mathrm{Mg}$ & $\mathrm{S}$ & $\mathrm{Zn}$ & $\mathrm{Fe}$ & $\mathrm{Mn}$ & $\mathrm{Cu}$ & $\mathrm{B}$ \\
\cline { 2 - 10 } Tratamentos $^{1}$ & --------------------- $\mathrm{g} \mathrm{kg}^{-1}$ & ------------------ & $------------\mathrm{mg} \mathrm{kg}^{-1}$ & ------------- \\
\hline B1 & 13,3 & 2,5 & 0,8 & 8,9 & 2,4 & 0,14 & 231 & 17480 & 157 & 53 & 8 \\
B2 & 13 & 2,9 & 1,3 & 8,2 & 2,1 & 0,17 & 247 & 17200 & 185 & 58 & 6 \\
B3 & 9,8 & 2,8 & 1,6 & 6,2 & 1,9 & 0,19 & 209 & 17280 & 184 & 43 & 6 \\
B4 & 10,5 & 2,3 & 2,7 & 4,0 & 1,7 & 0,11 & 183 & 12560 & 277 & 37 & 5 \\
B5 & 7,0 & 1,7 & 3,6 & 5,5 & 1,6 & 0,11 & 241 & 11040 & 322 & 28 & 16 \\
B6 & 11,2 & 2,3 & 1,2 & 5,5 & 1,8 & 0,14 & 225 & 15560 & 128 & 51 & 6 \\
B7 & 12,3 & 2,7 & 2,6 & 9,2 & 3,1 & 0,12 & 265 & 17000 & 178 & 65 & 12 \\
B8 & 10,5 & 2,7 & 3,1 & 7,7 & 2,6 & 0,14 & 240 & 15000 & 148 & 92 & 15 \\
B9 & 9,8 & 2,5 & 7,4 & 6,3 & 4,9 & 0,16 & 230 & 12080 & 158 & 64 & 24 \\
S10 & 8,8 & 1,6 & 1,2 & 8,3 & 4,1 & 0,06 & 44 & 9200 & 199 & 10 & 14 \\
\hline CAC & 4,6 & 1,1 & 6,5 & 2,1 & 1 & 0,06 & 44 & 506 & 492 & 8 & 6 \\
FC & 5,3 & 1,3 & 12,2 & 10,9 & 5,2 & 0,18 & 87 & 1130 & 78 & 50 & 50 \\
\hline
\end{tabular}

Experimento II

\begin{tabular}{cccccccccccc}
\hline \multirow{2}{*}{ Tratamentos $^{1}$} & $\mathrm{~N}$ & $\mathrm{P}$ & $\mathrm{K}$ & $\mathrm{Ca}$ & $\mathrm{Mg}$ & $\mathrm{S}$ & $\mathrm{Zn}$ & $\mathrm{Fe}$ & $\mathrm{Mn}$ & $\mathrm{Cu}$ & $\mathrm{B}$ \\
\cline { 2 - 10 } & -------------------- $\mathrm{g} \mathrm{kg}^{-1}$ & ------------------ & ------------ & $\mathrm{mg} \mathrm{kg}^{-1}$ & ------------ \\
\hline M1 & 2,1 & 1,1 & 2,2 & 6,1 & 2,6 & 0,07 & 52 & 32640 & 154 & 10 & 4 \\
M2 & 1,8 & 0,9 & 1,7 & 7,2 & 3,5 & 0,06 & 47 & 31520 & 118 & 7 & 4 \\
M3 & 1,8 & 0,9 & 1,4 & 9,3 & 3,4 & 0,06 & 40 & 32400 & 109 & 8 & 4 \\
M4 & 3,9 & 3,0 & 1,4 & 6,7 & 2,5 & 0,06 & 110 & 29160 & 138 & 22 & 7 \\
M5 & 2,5 & 1,6 & 1,4 & 13,0 & 2,2 & 0,06 & 69 & 34680 & 125 & 14 & 2 \\
M6 & 2,5 & 1,6 & 1,4 & 4,5 & 1,9 & 0,06 & 66 & 35280 & 109 & 16 & 5 \\
M7 & 3,2 & 3,0 & 1,8 & 19,1 & 2,3 & 0,07 & 94 & 33240 & 121 & 17 & 9 \\
M8 & 2,1 & 2,1 & 1,7 & 14,2 & 2,2 & 0,07 & 79 & 36160 & 128 & 14 & 6 \\
M9 & 2,5 & 1,3 & 1,3 & 4,9 & 1,3 & 0,07 & 61 & 37560 & 91 & 14 & 7 \\
S10 & 8,8 & 1,6 & 1,2 & 8,3 & 4,1 & 0,06 & 44 & 9200 & 199 & 10 & 14 \\
\hline TS & 0,4 & 0,5 & 0,8 & 11,4 & 2,2 & 0,06 & 39 & 37880 & 83 & 11 & 9 \\
EB & 4,6 & 1,6 & 4,5 & 12,7 & 5,1 & 0,05 & 68 & 12560 & 216 & 11 & 10 \\
CF & 7,7 & 4,6 & 2,4 & 14,2 & 5,7 & 0,06 & 256 & 7600 & 259 & 40 & 18 \\
EC & 7,7 & 4,8 & 4,3 & 35,7 & 4,8 & 0,09 & 219 & 9720 & 197 & 25 & 17 \\
\hline
\end{tabular}

Para o cálculo de quantidade de nutrientes da parte aérea das mudas, foi levado em consideração as concentrações dos nutrientes multiplicando-se por sua, respectiva massa.

Para determinação da concentração dos nutrientes presentes no substrato foi utilizada a 
mesma metodologia de determinação no tecido vegetal, conforme EMBRAPA (2009), realizada no LAFARSOL. Sendo que os substratos foram submetidos às análises antes do plantio das mudas.

\section{Resultados e Discussão}

Concentração e quantidade de nutrientes das mudas produzidas em substratos formulados com biossólido (experimento I)

Na Tabela 3 são apresentados os efeitos dos substratos formulados com biossólido na concentração e quantidade de nutrientes das mudas de $T$. grandis. A análise de variância revelou diferenças significativas entre os tratamentos, observada por meio do teste de Scott-Knott a 5\% de probabilidade.

Tabela 3. Concentração média e quantidade de nutrientes na parte aérea das mudas de Tectona grandis produzidas em substratos formulados com biossólido (experimento I).

Table 3. Mean concentration and amount of nutrientes in shoots of seedling of Tectona grandis produced on substrates formulated with biosolids (experiment I)

\begin{tabular}{|c|c|c|c|c|c|c|c|c|c|c|c|}
\hline \multicolumn{12}{|c|}{ Concentração de nutrientes (experimento I) } \\
\hline \multirow{2}{*}{ Tratamentos $^{2}$} & $\mathrm{~N}$ & $\mathrm{P}$ & $\mathrm{K}$ & $\mathrm{Ca}$ & $\mathrm{Mg}$ & $\mathrm{S}$ & $\mathrm{Zn}$ & $\mathrm{Fe}$ & $\mathrm{Mn}$ & $\mathrm{Cu}$ & $\mathrm{B}$ \\
\hline & \multicolumn{6}{|c|}{ 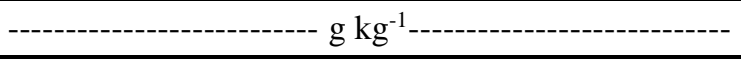 } & \multicolumn{5}{|c|}{ - } \\
\hline B1 & $13,1 \mathrm{c}^{1}$ & $3,1 \mathrm{~b}$ & $4,3 \mathrm{c}$ & $16,4 \mathrm{~b}$ & $3,1 \mathrm{e}$ & $1,3 \mathrm{a}$ & $85 d$ & $137 \mathrm{~b}$ & $71 \mathrm{c}$ & $11 \mathrm{a}$ & $23 \mathrm{c}$ \\
\hline B2 & $15,2 \mathrm{a}$ & $2,6 \mathrm{c}$ & $4,5 \mathrm{c}$ & $17,0 \mathrm{a}$ & $2,6 \mathrm{f}$ & $0,9 \mathrm{~b}$ & $110 \mathrm{~b}$ & $116 \mathrm{c}$ & $63 \mathrm{c}$ & $10 \mathrm{~b}$ & $20 \mathrm{c}$ \\
\hline B3 & $13,2 \mathrm{c}$ & $2,2 \mathrm{~d}$ & $4,6 \mathrm{c}$ & $13,4 \mathrm{e}$ & $2,5 \mathrm{~g}$ & $0,9 \mathrm{~b}$ & $88 \mathrm{~d}$ & $89 \mathrm{c}$ & $58 \mathrm{c}$ & $8 \mathrm{c}$ & $17 \mathrm{~d}$ \\
\hline B4 & $12,8 \mathrm{c}$ & $2,7 \mathrm{c}$ & $4,7 \mathrm{c}$ & $12,8 \mathrm{e}$ & $2,4 \mathrm{~g}$ & $0,9 \mathrm{~b}$ & $99 \mathrm{c}$ & $107 \mathrm{c}$ & $82 \mathrm{~b}$ & $10 \mathrm{~b}$ & $18 \mathrm{~d}$ \\
\hline B5 & $10,6 \mathrm{e}$ & $3,6 \mathrm{a}$ & $8,4 \mathrm{a}$ & $13,1 \mathrm{e}$ & $2,9 \mathrm{f}$ & $0,9 \mathrm{~b}$ & $71 \mathrm{e}$ & $105 \mathrm{c}$ & $78 \mathrm{~b}$ & $11 \mathrm{a}$ & $19 \mathrm{~d}$ \\
\hline B6 & $11,2 \mathrm{~d}$ & $3,2 \mathrm{~b}$ & $3,7 \mathrm{c}$ & $15,4 \mathrm{c}$ & $2,9 \mathrm{f}$ & $1,1 \mathrm{~b}$ & $123 \mathrm{a}$ & $185 \mathrm{a}$ & $104 \mathrm{a}$ & $10 \mathrm{~b}$ & $21 \mathrm{c}$ \\
\hline B7 & $7,7 \mathrm{~g}$ & $2,7 \mathrm{c}$ & $4,0 \mathrm{c}$ & $16,3 \mathrm{~b}$ & $3,8 \mathrm{~d}$ & $0,9 \mathrm{~b}$ & $129 \mathrm{a}$ & $202 \mathrm{a}$ & $94 \mathrm{a}$ & $6 \mathrm{~d}$ & $27 \mathrm{~b}$ \\
\hline B8 & $8,5 \mathrm{f}$ & $3,2 \mathrm{~b}$ & $3,6 \mathrm{c}$ & $17,5 \mathrm{a}$ & $4,2 \mathrm{c}$ & $1,1 \mathrm{a}$ & $87 \mathrm{~d}$ & $151 \mathrm{~b}$ & $51 \mathrm{c}$ & $8 \mathrm{c}$ & $21 \mathrm{c}$ \\
\hline B9 & $8,5 \mathrm{f}$ & $2,9 \mathrm{~b}$ & $4,1 \mathrm{c}$ & $16,8 \mathrm{a}$ & $5,5 \mathrm{~b}$ & $0,8 \mathrm{~b}$ & $58 \mathrm{f}$ & $144 \mathrm{~b}$ & $59 \mathrm{c}$ & $8 \mathrm{c}$ & $22 c$ \\
\hline S10 & $13,8 \mathrm{~b}$ & $2,1 \mathrm{~d}$ & $6,9 \mathrm{~b}$ & $14,1 \mathrm{~d}$ & $7,4 \mathrm{a}$ & $1,4 \mathrm{a}$ & $54 \mathrm{f}$ & $161 \mathrm{~b}$ & $49 \mathrm{c}$ & $2,83 \mathrm{e}$ & $30 \mathrm{a}$ \\
\hline $\mathrm{CV}(\%)$ & 2,84 & 5,82 & 11,57 & 2,47 & 2,85 & 13,76 & 6,18 & 9,84 & 14,49 & 3,35 & 6,99 \\
\hline \multicolumn{12}{|c|}{ Quantidade de nutrientes (experimento I) } \\
\hline \multirow{2}{*}{ Tratamentos $^{2}$} & $\mathrm{~N}$ & $\mathrm{P}$ & $\mathrm{K}$ & $\mathrm{Ca}$ & $\mathrm{Mg}$ & S & $\mathrm{Zn}$ & $\mathrm{Fe}$ & $\mathrm{Mn}$ & $\mathrm{Cu}$ & B \\
\hline & \multicolumn{6}{|c|}{ 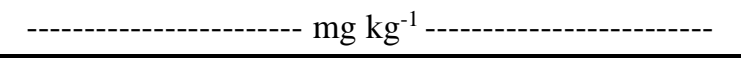 } & \multicolumn{5}{|c|}{ - } \\
\hline B1 & $62,6 \mathrm{~d}^{1}$ & $15,1 \mathrm{c}$ & $20,8 \mathrm{c}$ & $78,4 \mathrm{e}$ & $15,0 \mathrm{e}$ & $6,2 \mathrm{a}$ & 4094 e & $6586 \mathrm{c}$ & $3417 \mathrm{~d}$ & $539 \mathrm{~d}$ & $1130 \mathrm{c}$ \\
\hline B2 & $120,1 \mathrm{a}$ & $20,3 \mathrm{a}$ & $35,4 \mathrm{a}$ & $134,8 \mathrm{a}$ & $21,7 \mathrm{a}$ & $7,2 \mathrm{a}$ & 8679 a & $9207 \mathrm{a}$ & $5019 \mathrm{~b}$ & $814 \mathrm{a}$ & $1647 \mathrm{a}$ \\
\hline B3 & $99,9 \mathrm{~b}$ & $16,9 \mathrm{~b}$ & 34,9 a & $101,7 \mathrm{~b}$ & $18,7 \mathrm{~b}$ & $6,5 \mathrm{a}$ & $6685 \mathrm{~b}$ & $6732 c$ & $4409 \mathrm{c}$ & $652 \mathrm{~b}$ & $1296 \mathrm{~b}$ \\
\hline B4 & $77,1 \mathrm{c}$ & $16,1 \mathrm{~b}$ & $28,4 \mathrm{~b}$ & $76,7 \mathrm{e}$ & $14,7 \mathrm{e}$ & $5,7 \mathrm{a}$ & $5950 \mathrm{c}$ & $6417 c$ & $4954 \mathrm{~b}$ & $644 b$ & $1094 \mathrm{c}$ \\
\hline B5 & $25,2 \mathrm{~h}$ & $08,5 \mathrm{f}$ & $19,9 \mathrm{c}$ & $31,1 \mathrm{~g}$ & $6,8 \mathrm{f}$ & $2,1 \mathrm{c}$ & $1679 \mathrm{f}$ & $2483 \mathrm{e}$ & $1842 \mathrm{e}$ & $268 \mathrm{~g}$ & $454 \mathrm{e}$ \\
\hline B6 & $63,8 \mathrm{~d}$ & $18,0 \mathrm{~b}$ & $20,9 \mathrm{c}$ & $87,5 \mathrm{~d}$ & $16,3 \mathrm{~d}$ & $6,0 \mathrm{a}$ & $7022 \mathrm{c}$ & $10515 \mathrm{a}$ & 5929 a & $609 c$ & $1241 b$ \\
\hline B7 & $36,8 \mathrm{f}$ & $12,7 \mathrm{~d}$ & $19,1 \mathrm{c}$ & $78,1 \mathrm{e}$ & $17,9 \mathrm{c}$ & $4,2 \mathrm{~b}$ & $6194 b$ & $9677 \mathrm{a}$ & $4485 \mathrm{c}$ & $331 \mathrm{f}$ & $1308 \mathrm{~b}$ \\
\hline B8 & $45,0 \mathrm{e}$ & $16,7 \mathrm{~b}$ & $18,8 \mathrm{c}$ & $92,4 \mathrm{c}$ & $22,3 \mathrm{a}$ & $6,0 \mathrm{a}$ & $4605 \mathrm{~d}$ & 7977 b & $2716 \mathrm{e}$ & $429 \mathrm{e}$ & $1154 \mathrm{c}$ \\
\hline B9 & $30,0 \mathrm{~g}$ & $10,3 \mathrm{e}$ & $14,3 \mathrm{~d}$ & $59,2 \mathrm{f}$ & $19,4 \mathrm{~b}$ & $2,9 \mathrm{c}$ & $2056 \mathrm{f}$ & $5094 \mathrm{~d}$ & $2082 \mathrm{e}$ & $291 \mathrm{~g}$ & $794 \mathrm{~d}$ \\
\hline $\mathrm{S} 10$ & $13,7 \mathrm{i}$ & $02,1 \mathrm{~g}$ & $06,9 \mathrm{e}$ & $14,0 \mathrm{~h}$ & $7,3 \mathrm{f}$ & $1,4 \mathrm{c}$ & $539 \mathrm{~g}$ & $1595 \mathrm{e}$ & $490 \mathrm{f}$ & $28 \mathrm{~h}$ & $299 \mathrm{f}$ \\
\hline $\mathrm{CV}(\%)$ & 2,98 & 6,28 & 9,91 & 2,03 & 2,96 & 17,69 & 5,58 & 10,17 & 15,4 & 4,29 & 6,28 \\
\hline
\end{tabular}

1Médias seguidas da mesma letra, na coluna, não diferem estatisticamente entre si pelo teste de Scott-Knott $(\mathrm{P}>0,05)$. 
Experimento I: ${ }^{2} \mathrm{BIO}$ (biossólido), CAC (casca de arroz carbonizada), FC (fibra de coco triturada).

A parte aérea das mudas apresentou maiores concentrações, para a maioria dos nutrientes, nos tratamentos formulados com BIO do que no tratamento S10, indicando a importância do biossólido não só para a quantidade como também para as concentrações dos nutrientes na planta.

A parte aérea apresentou concentrações de $\mathrm{N}$ entre 7,7 a $15,17 \mathrm{~g} \mathrm{~kg}^{-1}$, sendo o maior no tratamento $\mathrm{B} 2(80 \% \mathrm{BIO}+20 \% \mathrm{CAC})$ e o menor no B7 $(60 \% \mathrm{BIO}+40 \% \mathrm{FC})$. O tratamento S10 (substrato comercial florestal) apresentou média igual a $13,77 \mathrm{~g} \mathrm{~kg}^{-1}$, sendo estatisticamente inferior apenas ao tratamento B2. D’ávilla (2008), obteve concentrações foliares menores que neste estudo, ao trabalhar com doses de $\mathrm{N}$ aplicadas na fertirrigação do substrato, encontrou valores próximos de $3 \mathrm{~g} \mathrm{~kg}^{-1}$ na maior adubação, em mudas de eucalipto, aos 42 dias após estaqueamento. Em contrapartida, Silva et al. (2007), encontraram até $24,61 \mathrm{~g} \mathrm{~kg}^{-1}$ de $\mathrm{N}$ na parte aérea de mogno (Swietenia macrophylla King.), 120 dias após a repicagem.

$\mathrm{O}$ tratamento B5 $(20 \% \mathrm{BIO}+80 \% \mathrm{CAC})$ apresentou as maiores concentrações médias de $\mathrm{P}$ e K, diferenciando-se dos demais. As maiores concentrações de K no substrato, fornecidos pela casca de arroz carbonizada ou fibra de coco triturada, influenciaram na quantidade deste nutriente na parte aérea das mudas. Entretanto, o $\mathrm{P}$ nos substratos, não apresentou a mesma influência. Este resultado também foi constatado por Trigueiro e Guerrini (2003), em mudas de eucalipto.

As concentrações de $\mathrm{Ca}, \mathrm{Mg}$ e $\mathrm{S}$ apresentaram diferenças significativas entre os tratamentos, no entanto, não houve um padrão de resposta aos nutrientes.

$\mathrm{Na}$ produção de mudas de Acacia mangium, Fonseca (2010), usando como substrato composto de lixo urbano encontrou concentrações na parte aérea das mudas iguais $17,26 \mathrm{~g} \mathrm{~kg}^{-1}$ para $\mathrm{N}, 2,05 \mathrm{~g} \mathrm{~kg}^{-1}$ para $\mathrm{P}, 3,87 \mathrm{~g} \mathrm{~kg}^{-}$ ${ }^{1}$ para $\mathrm{K}, 15,13 \mathrm{~g} \mathrm{~kg}^{-1}$ para Ca e $1,55 \mathrm{~g} \mathrm{~kg}^{-1}$ para $\mathrm{Mg}$, em compostos com $70 \%$ do composto, 120 dias após a semeadura. O mesmo autor também encontrou valores próximos ao trabalhem com
Mimosa artemisiana, com o mesmo substrato e com a mesma idade: $17,17 \mathrm{~g} \mathrm{~kg}^{-1}$ de $\mathrm{N}, 3,95 \mathrm{~g}$ $\mathrm{kg}^{-1}$ de $\mathrm{P}, 8,5 \mathrm{~g} \mathrm{~kg}^{-1}$ de $\mathrm{K}, 20,91 \mathrm{~g} \mathrm{~kg}^{-1}$ de $\mathrm{Ca}$ e $2,58 \mathrm{~g} \mathrm{~kg}^{-1}$ de $\mathrm{Mg}$.

Com relação aos micronutrientes, vale ressaltar a influência do substrato na adição de $\mathrm{Zn}, \mathrm{Fe}$ e $\mathrm{Mn}$ na parte aérea, onde as concentrações destes micronutrientes presentes no BIO, CAC e FC, contribuíram para tal ganho. Os tratamentos B6 $(80 \% \mathrm{BIO}+20 \% \mathrm{FC})$ e $\mathrm{B} 7$ $(40 \% \mathrm{BIO}+60 \% \mathrm{FC})$ apresentaram as maiores médias das concentrações de $\mathrm{Zn}$, Fe e Mn, diferenciando-se dos demais tratamentos. As concentrações mais baixas de $\mathrm{Zn}$ e $\mathrm{Mn}$, foram alcançadas no tratamento S10 (substrato comercial florestal).

Com relação à quantidade de macronutrientes (Tabela 3), a maior média foi obtida no tratamento $\mathrm{B} 2(80 \% \mathrm{BIO}+20 \% \mathrm{CAC})$, seguido dos tratamentos $\mathrm{B} 3(60 \% \mathrm{BIO}+40 \% \mathrm{CAC})$ e $\mathrm{B} 6$ $(80 \% \mathrm{BIO}+20 \% \mathrm{FC})$. O fato de estes substratos serem compostos na sua maioria por biossólido, contribuiu para uma maior quantidade dos nutrientes. Para os micronutrientes, a maior quantidade foi encontrada no tratamento B2 $(80 \%$ BIO + 20\%CAC), com exceção do Mn, que apresentou maior quantidade no tratamento B5 $(20 \% \mathrm{BIO}+80 \% \mathrm{CAC})$.

Para todos os nutrientes, o tratamento S10 (substrato comercial florestal) apresentou a menor quantidade. Fato contrário ao ocorrido com Trigueiro e Guerrini (2003), que testando biossólido para produção de mudas de eucalipto, encontraram maiores quantidades de nutrientes nas mudas produzidas em substrato comercial florestal.

Comparando as concentrações de nutrientes no substrato com os teores de nutrientes na parte aérea das mudas é possível observar, na Tabela 4, que a correlação não foi significativa para a maioria dos nutrientes, sendo que apenas para $\mathrm{Ca}$ $(\mathrm{R}=0,64 * *), \mathrm{Mg}\left(\mathrm{R}=0,87^{* *}\right)$ e $\mathrm{Zn}\left(\mathrm{R}=0,53^{*}\right)$ os níveis desses nutrientes na parte aérea apresentaram correlação positiva com as concentrações totais dos mesmos nutrientes nos substratos. A correlação foi significativa e negativa entre a concentração de $\mathrm{S}$ na parte aérea 
no substrato $\left(\mathrm{R}=-0,59^{*}\right)$ (Tabela 4$)$.

Tabela 4. Correlação entre as características químicas dos substratos e as concentrações de nutrientes na parte aérea das mudas de Tectona grandis produzidas nos substratos formulados com biossólido (experimento I).

Table 4. Correlations between the chemical characteristics of the substrates and nutrient concentrations in shoots of seedling of Tectona grandis produced on substrates formulated with biosolids (experiment I).

\begin{tabular}{|c|c|c|c|c|c|c|c|c|c|c|c|}
\hline & $\mathrm{N}$ & $\mathrm{P}$ & $\bar{K}$ & $\mathrm{Ca}$ & $\mathrm{Mg}$ & 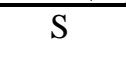 & $\mathrm{Zn}$ & $\mathrm{Fe}$ & $\mathrm{Mn}$ & $\mathrm{Cu}$ & $\bar{B}$ \\
\hline $\mathrm{TN}$ & $0,15^{\mathrm{ns}}$ & $-0,10^{\mathrm{ns}}$ & $-0,64 * *$ & $-0,04^{\mathrm{ns}}$ & $-0,34^{\mathrm{ns}}$ & $0,02^{\mathrm{ns}}$ & $-0,44^{*}$ & $0,05^{\mathrm{ns}}$ & $0,16^{\mathrm{ns}}$ & $-0,56^{*}$ & $-0,64 * *$ \\
\hline TP & $-0,13^{\text {ns }}$ & $-0,16^{\mathrm{ns}}$ & $0,29^{\text {ns }}$ & $-0,23^{\text {ns }}$ & $-0,29^{\text {ns }}$ & $0,03^{\text {ns }}$ & $0,57 *$ & $-0,03^{\text {ns }}$ & $0,17^{\mathrm{ns}}$ & $0,31^{\mathrm{ns}}$ & $0,27^{\mathrm{ns}}$ \\
\hline TK & $-0,74^{*}$ & $-0,82 * *$ & $-0,02^{\mathrm{ns}}$ & $-0,16^{\mathrm{ns}}$ & $-0,07^{\mathrm{ns}}$ & $-0,57 *$ & $-0,43^{*}$ & $-0,68 * *$ & $0,78 * *$ & $-0,77 * *$ & $0,22^{\mathrm{ns}}$ \\
\hline $\mathrm{TCa}$ & $0,62 * *$ & $0,57^{*}$ & $0,16^{\mathrm{ns}}$ & $0,64 * *$ & $0,39^{\text {ns }}$ & $0,35^{\mathrm{ns}}$ & $0,44^{*}$ & $0,45^{*}$ & $-0,73 * *$ & $0,80 * *$ & $0,28^{\mathrm{ns}}$ \\
\hline $\mathrm{TMg}$ & $-0,30^{\text {ns }}$ & $-0,45^{*}$ & $0,26^{\mathrm{ns}}$ & $0,37^{\mathrm{ns}}$ & $0,87 * *$ & $-0,55^{*}$ & $-0,68^{* *}$ & $-0,62 * *$ & $-0,21^{\mathrm{ns}}$ & $-0,21^{\mathrm{ns}}$ & $0,64 * *$ \\
\hline TS & $0,06^{\mathrm{ns}}$ & $-0,44^{*}$ & $-0,53^{*}$ & $0,42 *$ & $0,17^{\mathrm{ns}}$ & $-0,59 *$ & $-0,66^{* *}$ & $-0,23^{\mathrm{ns}}$ & $-0,22^{\mathrm{ns}}$ & $-0,29^{\mathrm{ns}}$ & $-0,08^{\mathrm{ns}}$ \\
\hline $\mathrm{TZn}$ & $0,63 * *$ & $0,54 *$ & $-0,41^{*}$ & $0,07^{\mathrm{ns}}$ & $-0,51 *$ & $0,27^{\mathrm{ns}}$ & $0,53 *$ & $0,70 * *$ & $-0,25^{\mathrm{ns}}$ & $0,38^{\mathrm{ns}}$ & $-0,63 * *$ \\
\hline $\mathrm{TFe}$ & $0,31^{\mathrm{ns}}$ & $-0,02^{\text {ns }}$ & $-0,03^{\text {ns }}$ & $0,46^{*}$ & $0,41 *$ & $-0,34^{\text {ns }}$ & $0,00^{\mathrm{ns}}$ & $0,06^{\mathrm{ns}}$ & $-0,55^{*}$ & $0,28^{\mathrm{ns}}$ & $0,20^{\mathrm{ns}}$ \\
\hline TMn & $0,24^{\mathrm{ns}}$ & $-0,01^{\mathrm{ns}}$ & $-0,17^{\mathrm{ns}}$ & $-0,27^{\mathrm{ns}}$ & $-0,44 *$ & $-0,06^{\text {ns }}$ & $0,40 *$ & $0,25^{\mathrm{ns}}$ & $0,07^{\mathrm{ns}}$ & $-0,01^{\text {ns }}$ & $-0,37^{\mathrm{ns}}$ \\
\hline $\mathrm{TCu}$ & $0,23^{\mathrm{ns}}$ & $0,26^{\mathrm{ns}}$ & $-0,02^{\mathrm{ns}}$ & $-0,44^{*}$ & $-0,69 * *$ & $0,48^{*}$ & $0,69^{*}$ & $0,40 *$ & $0,21^{\mathrm{ns}}$ & $0,22^{\mathrm{ns}}$ & $-0,36^{\mathrm{ns}}$ \\
\hline TB & $0,14^{\mathrm{ns}}$ & $-0,31^{\mathrm{ns}}$ & $-0,12^{\mathrm{ns}}$ & $0,70^{* *}$ & $0,65^{* *}$ & $-0,62 * *$ & $-0,47^{*}$ & $-0,24^{\mathrm{ns}}$ & $-0,33^{\mathrm{ns}}$ & $-0,13^{\mathrm{ns}}$ & $0,32^{\mathrm{ns}}$ \\
\hline
\end{tabular}

Teste F: ** significativo ao nível de $1 \%$ de probabilidade; * significativo ao nível de $5 \%$ de probabilidade; ${ }^{\text {ns }}$ não significativo $(\mathrm{p}>0,05)$.

A correlação entre as concentrações totais no substrato com biossólido e na parte aérea pode indicar, que devido ao efeito de diluição, apenas o $\mathrm{Ca}, \mathrm{Mg}$ e $\mathrm{Zn}$ foram significativos com uma correlação positiva. Para o S, cuja correlação foi significativa, porém negativa, pode ter ocorrido lixiviação do $\mathrm{SO}_{4}{ }^{-2}$, uma vez que este apresenta boa mobilidade no solo, podendo ser perdido por lixiviação (ALVAREZ et al., 2007).
Na Tabela 5, é possível observar que a correlação entre cada nutriente no substrato e a quantidade deste na parte aérea foi significativa e positiva para $\mathrm{N}(\mathrm{R}=0,53 *), \mathrm{P}(\mathrm{R}=0,80 * *), \mathrm{S}$ $\left(\mathrm{R}=0,69^{* *}\right), \mathrm{Zn}\left(\mathrm{R}=0,51^{*}\right)$ e $\mathrm{Fe}\left(\mathrm{R}=0,81^{* *}\right)$; não significativa para $\mathrm{K}\left(\mathrm{R}=-0,29^{\mathrm{ns}}\right), \mathrm{Ca}(\mathrm{R}=$ $\left.0,09^{\text {ns }}\right), M g\left(R=0,02^{\text {ns }}\right), M n\left(R=-0,23^{\text {ns }}\right)$ e $\mathrm{Cu}$ $\left(\mathrm{R}=0,32^{\mathrm{ns}}\right)$; e significativa, porém negativa para $\mathrm{B}(\mathrm{R}=-0,60 * *)$.

Tabela 5. Correlação entre as características químicas dos substratos e as quantidades de nutrientes na parte aérea das mudas de Tectona grandis produzidas nos substratos formulados com biossólido (experimento I).

Table 5. Correlations between chemical characteristics of the substrates and the amounts of nutrientes in shoots of seedlings of Tectona grandis produced on substrates formulated with biosolid (experiment I).

\begin{tabular}{cccccccccccc}
\hline & $\mathrm{N}$ & $\mathrm{P}$ & $\mathrm{K}$ & $\mathrm{Ca}$ & $\mathrm{Mg}$ & $\mathrm{S}$ & $\mathrm{Zn}$ & $\mathrm{Fe}$ & $\mathrm{Mn}$ & $\mathrm{Cu}$ & $\mathrm{B}$ \\
\hline $\mathrm{AN}$ & $0,53^{*}$ & $0,67^{* *}$ & $-0,42^{*}$ & $-0,12^{\text {ns }}$ & $-0,56^{*}$ & $0,69^{* *}$ & $0,32^{\text {ns }}$ & $0,68^{* *}$ & $-0,13^{\text {ns }}$ & $0,18^{\text {ns }}$ & $-0,75^{* *}$ \\
$\mathrm{AP}$ & $0,63^{* *}$ & $0,80^{* *}$ & $-0,25^{\text {ns }}$ & $-0,13^{\text {ns }}$ & $-0,59^{*}$ & $0,74^{* *}$ & $0,65^{* *}$ & $0,80^{* *}$ & $-0,32^{\text {ns }}$ & $0,59^{*}$ & $-0,61^{* *}$ \\
$\mathrm{AK}$ & $0,35^{\text {ns }}$ & $0,63^{* *}$ & $-0,29^{\text {ns }}$ & $-0,25^{\text {ns }}$ & $-0,68^{* *}$ & $0,70^{* *}$ & $0,46^{*}$ & $0,64^{* *}$ & $0,11^{\text {ns }}$ & $0,16^{\text {ns }}$ & $-0,68^{* *}$ \\
$\mathrm{ACa}$ & $0,70^{* *}$ & $0,90^{* *}$ & $-0,25^{\text {ns }}$ & $0,09^{\text {ns }}$ & $-0,42^{*}$ & $0,79^{* *}$ & $0,59^{*}$ & $0,84^{* *}$ & $-0,42^{*}$ & $0,61^{* *}$ & $-0,55^{*}$ \\
$\mathrm{AMg}$ & $0,62^{* *}$ & $0,95^{* *}$ & $0,14^{\text {ns }}$ & $0,18^{\text {ns }}$ & $0,02^{\text {ns }}$ & $0,76^{* *}$ & $0,58^{*}$ & $0,69^{* *}$ & $-0,63^{* *}$ & $0,86^{* *}$ & $-0,11^{\text {ns }}$ \\
$\mathrm{AS}$ & $0,71^{* *}$ & $0,79^{* *}$ & $-0,43^{*}$ & $0,01^{\text {ns }}$ & $-0,57^{*}$ & $0,69^{* *}$ & $0,48^{*}$ & $0,84^{* *}$ & $-0,39^{\text {ns }}$ & $0,51^{*}$ & $-0,72^{* *}$ \\
$\mathrm{AZn}$ & $0,66^{* *}$ & $0,76^{* *}$ & $-0,41^{*}$ & $-0,02^{\text {ns }}$ & $-0,56^{*}$ & $0,60^{* *}$ & $0,51^{*}$ & $0,80^{* *}$ & $-0,29^{\text {ns }}$ & $0,40^{*}$ & $-0,74^{* *}$ \\
$\mathrm{AFe}$ & $0,75^{* *}$ & $0,77^{* *}$ & $-0,25^{\text {ns }}$ & $0,13^{\text {ns }}$ & $-0,33^{\text {ns }}$ & $0,54^{*}$ & $0,63^{* *}$ & $0,81^{* *}$ & $-0,57^{*}$ & $0,67^{* *}$ & $-0,48^{*}$ \\
$\mathrm{AMn}$ & $0,61^{* *}$ & $0,62^{* *}$ & $-0,35^{\text {ns }}$ & $-0,22^{\text {ns }}$ & $-0,60^{* *}$ & $0,52^{*}$ & $0,52^{*}$ & $0,72^{* *}$ & $-0,23^{\text {ns }}$ & $0,31^{\text {ns }}$ & $-0,74^{* *}$ \\
$\mathrm{ACu}$ & $0,58^{*}$ & $0,69^{* *}$ & $-0,34^{\text {ns }}$ & $-0,22^{\text {ns }}$ & $-0,65^{* *}$ & $0,70^{* *}$ & $0,50^{*}$ & $0,72^{* *}$ & $-0,15^{\text {ns }}$ & $0,32^{\text {ns }}$ & $-0,74^{* *}$ \\
$\mathrm{AB}$ & $0,79^{* *}$ & $0,91^{* *}$ & $-0,30^{\text {ns }}$ & $0,15^{\text {ns }}$ & $-0,41^{*}$ & $0,71^{* *}$ & $0,62^{* *}$ & $0,90^{* *}$ & $-0,44^{*}$ & $0,61^{* *}$ & $-0,60^{* *}$ \\
\hline
\end{tabular}

Teste F: $* *$ significativo ao nível de $1 \%$ de probabilidade; * significativo ao nível de $5 \%$ de probabilidade; ${ }^{\text {ns }}$ não significativo 
$(\mathrm{p}>0,05)$.

As correlações entre as quantidades e as concentrações de nutrientes na parte aérea indicaram que pode ter ocorrido perda por lixiviação de alguns nutrientes, principalmente o B (correlação significativa e negativa), que apresentaram altas concentrações nos substratos (ALVAREZ V. et al., 1999), no entanto, a adsorção de $\mathrm{B}$ ocorre até determinado ponto crítico, a partir do qual a adsorção é baixa (AZEVEDO, 2001), e a partir deste ponto, somente terá como consequência maior perda do nutriente por lixiviação (ROSOLEM e
BÍSCARO, 2007).

Concentrações e quantidades de nutrientes das mudas produzidas em substratos formulados com estercos de animais (experimento II)

A concentração e a quantidade de nutrientes das mudas produzidas nos substratos com estercos de animais estão presentes nas Tabelas 6. A análise de variância mostrou diferenças significativas entre os tratamentos, ressaltada pelo teste de Scott-Knott a 5\% de probabilidade.

Tabela 6. Concentrações médias e quantidades de nutrientes na parte aérea das mudas de Tectona grandis produzidas em substratos formulados com esterco de animais (experimento II).

Table 6. Mean concentrations and amounts of nutrients in shoots of seedlings of Tectona grandis produced on substrates formulated with animal manure (experiment II).

\section{Concentração de nutrientes (experimento II)}

\begin{tabular}{|c|c|c|c|c|c|c|c|c|c|c|c|}
\hline \multirow{2}{*}{ Tratamentos ${ }^{2}$} & $\mathrm{~N}$ & $\mathrm{P}$ & $\mathrm{K}$ & $\mathrm{Ca}$ & $\mathrm{Mg}$ & $S$ & $\mathrm{Zn}$ & $\mathrm{Fe}$ & $\mathrm{Mn}$ & $\mathrm{Cu}$ & B \\
\hline & \multicolumn{6}{|c|}{ - } & \multicolumn{5}{|c|}{ - } \\
\hline M1 & $11,32 \mathrm{c}^{1}$ & $6,31 \mathrm{~b}$ & $8,87 \mathrm{a}$ & $8,56 \mathrm{c}$ & $3,01 \mathrm{f}$ & $1,58 \mathrm{~b}$ & $44,69 \mathrm{c}$ & $159,24 \mathrm{~b}$ & $32,78 \mathrm{c}$ & $9,53 \mathrm{c}$ & $51,85 \mathrm{c}$ \\
\hline M2 & $10,85 \mathrm{c}$ & $7,11 \mathrm{~b}$ & $7,80 \mathrm{~b}$ & $12,79 \mathrm{~b}$ & $3,86 \mathrm{e}$ & $1,76 \mathrm{a}$ & $50,53 \mathrm{~b}$ & $157,23 \mathrm{~b}$ & $37,73 \mathrm{~b}$ & $9,55 \mathrm{c}$ & $54,84 \mathrm{c}$ \\
\hline M3 & $9,45 \mathrm{c}$ & $5,39 \mathrm{c}$ & $7,03 \mathrm{c}$ & $14,58 \mathrm{a}$ & $3,40 \mathrm{f}$ & $1,56 \mathrm{~b}$ & $63,01 \mathrm{a}$ & $172,65 \mathrm{~b}$ & $46,22 \mathrm{a}$ & $8,58 \mathrm{~d}$ & $50,17 \mathrm{c}$ \\
\hline M4 & $10,85 \mathrm{c}$ & $7,58 \mathrm{~b}$ & $5,17 \mathrm{~d}$ & $14,92 \mathrm{a}$ & $6,62 \mathrm{~b}$ & $1,18 \mathrm{c}$ & 61,36 a & $144,42 \mathrm{~b}$ & $39,50 \mathrm{~b}$ & $10,57 \mathrm{~b}$ & $38,50 \mathrm{~d}$ \\
\hline M5 & $10,62 \mathrm{c}$ & $7,30 \mathrm{~b}$ & $5,63 \mathrm{~d}$ & $15,08 \mathrm{a}$ & $5,27 \mathrm{c}$ & $1,54 \mathrm{~b}$ & $45,88 \mathrm{c}$ & $144,17 \mathrm{~b}$ & $47,71 \mathrm{a}$ & $9,34 \mathrm{c}$ & $49,22 \mathrm{c}$ \\
\hline M6 & $10,50 \mathrm{c}$ & $8,54 \mathrm{a}$ & $6,93 \mathrm{c}$ & $14,73 \mathrm{a}$ & $4,50 \mathrm{~d}$ & $1,52 \mathrm{~b}$ & $51,52 \mathrm{~b}$ & $150,97 \mathrm{~b}$ & $37,97 \mathrm{~b}$ & $9,91 \mathrm{c}$ & $51,98 \mathrm{c}$ \\
\hline M7 & $12,60 \mathrm{~b}$ & $9,01 \mathrm{a}$ & $9,60 \mathrm{a}$ & $12,36 \mathrm{~b}$ & $3,96 \mathrm{e}$ & $1,92 \mathrm{a}$ & $60,28 \mathrm{a}$ & $164,57 \mathrm{~b}$ & $25,28 \mathrm{~d}$ & $11,49 \mathrm{a}$ & $63,69 \mathrm{a}$ \\
\hline M8 & $10,85 \mathrm{c}$ & $8,97 \mathrm{a}$ & $8,03 \mathrm{~b}$ & $14,39 \mathrm{c}$ & $4,62 \mathrm{~d}$ & $1,81 \mathrm{a}$ & $53,18 \mathrm{~b}$ & $160,22 \mathrm{~b}$ & $38,51 \mathrm{~b}$ & $10,75 \mathrm{~b}$ & $60,75 \mathrm{a}$ \\
\hline M9 & $10,15 \mathrm{c}$ & $9,44 \mathrm{a}$ & $7,60 \mathrm{~b}$ & $15,60 \mathrm{a}$ & $3,70 \mathrm{e}$ & $1,59 \mathrm{~b}$ & $60,20 \mathrm{a}$ & $204,70 \mathrm{a}$ & $47,08 \mathrm{a}$ & $8,97 \mathrm{~d}$ & $57,11 \mathrm{~b}$ \\
\hline S10 & $13,77 \mathrm{a}$ & $2,13 \mathrm{~d}$ & $6,93 \mathrm{c}$ & $14,12 \mathrm{a}$ & $7,40 \mathrm{a}$ & $1,42 \mathrm{~b}$ & $54,38 \mathrm{~b}$ & $160,80 \mathrm{~b}$ & $49,44 \mathrm{a}$ & $2,83 \mathrm{e}$ & $30,18 \mathrm{e}$ \\
\hline $\mathrm{CV}(\%)$ & 5,27 & 8,09 & 9,91 & 7,69 & 5,37 & 7,45 & 5,36 & 7,79 & 6,08 & 4,78 & 4,91 \\
\hline \multicolumn{12}{|c|}{ Quantidade de nutrientes (experimento II) } \\
\hline \multirow{2}{*}{ Tratamentos ${ }^{2}$} & $\mathrm{~N}$ & $\mathrm{P}$ & $\mathrm{K}$ & $\mathrm{Ca}$ & $\mathrm{Mg}$ & $\mathrm{S}$ & $\mathrm{Zn}$ & $\mathrm{Fe}$ & $\mathrm{Mn}$ & $\mathrm{Cu}$ & $\mathrm{B}$ \\
\hline & \multicolumn{6}{|c|}{ 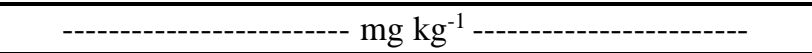 } & \multicolumn{5}{|c|}{ 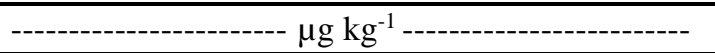 } \\
\hline M1 & $23,4 b^{1}$ & $13,1 \mathrm{c}$ & $18,4 \mathrm{~b}$ & $17,7 \mathrm{e}$ & $6,2 \mathrm{e}$ & $3,3 \mathrm{~b}$ & $925 \mathrm{~b}$ & $3297 \mathrm{~b}$ & $679 d$ & $197 \mathrm{c}$ & $1074 \mathrm{c}$ \\
\hline M2 & $21,8 \mathrm{c}$ & $14,3 \mathrm{c}$ & $15,7 \mathrm{c}$ & $25,7 \mathrm{~d}$ & $7,8 \mathrm{~d}$ & $3,5 \mathrm{~b}$ & $1015 \mathrm{~b}$ & $3159 \mathrm{~b}$ & $758 \mathrm{c}$ & $192 \mathrm{c}$ & $1102 \mathrm{c}$ \\
\hline M3 & $11,3 \mathrm{e}$ & $6,5 \mathrm{e}$ & $08,4 \mathrm{e}$ & $17,5 \mathrm{e}$ & $4,1 \mathrm{f}$ & $1,9 \mathrm{c}$ & $756 c$ & 2072 c & $555 \mathrm{e}$ & $103 \mathrm{e}$ & $602 \mathrm{e}$ \\
\hline M4 & $41,9 \mathrm{a}$ & $29,3 \mathrm{a}$ & $19,9 \mathrm{a}$ & 57,6 a & $25,6 \mathrm{a}$ & $4,6 \mathrm{a}$ & 2369 a & $5575 \mathrm{a}$ & $1525 \mathrm{a}$ & 408 a & $1486 \mathrm{a}$ \\
\hline M5 & $24,5 \mathrm{~b}$ & $16,9 \mathrm{~b}$ & $13,0 \mathrm{~d}$ & $34,9 \mathrm{~b}$ & $12,2 \mathrm{~b}$ & $3,6 \mathrm{~b}$ & $1061 \mathrm{~b}$ & 3332 b & $1103 \mathrm{~b}$ & $216 \mathrm{~b}$ & $1138 \mathrm{~b}$ \\
\hline M6 & $13,5 \mathrm{~d}$ & $10,9 \mathrm{~d}$ & $08,9 \mathrm{e}$ & $18,9 \mathrm{e}$ & $5,8 \mathrm{e}$ & $1,9 \mathrm{c}$ & $660 \mathrm{c}$ & $1934 \mathrm{c}$ & $487 \mathrm{e}$ & $127 \mathrm{~d}$ & $666 \mathrm{~d}$ \\
\hline M7 & $13,2 \mathrm{~d}$ & $9,5 \mathrm{~d}$ & $10,1 \mathrm{e}$ & $13,0 \mathrm{f}$ & $4,2 \mathrm{f}$ & $2,0 \mathrm{c}$ & $633 c$ & $1729 \mathrm{~d}$ & $266 \mathrm{f}$ & $121 \mathrm{~d}$ & $669 \mathrm{~d}$ \\
\hline M8 & $22,7 \mathrm{c}$ & $18,8 \mathrm{~b}$ & $16,8 \mathrm{~b}$ & $30,1 \mathrm{c}$ & $9,7 \mathrm{c}$ & $3,8 \mathrm{~b}$ & $1112 \mathrm{~b}$ & $3350 \mathrm{~b}$ & $805 \mathrm{c}$ & $225 \mathrm{~b}$ & $1270 \mathrm{c}$ \\
\hline M9 & $10,2 \mathrm{e}$ & $9,5 \mathrm{~d}$ & $07,7 \mathrm{e}$ & $15,7 \mathrm{f}$ & $3,7 \mathrm{f}$ & $1,6 \mathrm{~d}$ & $606 \mathrm{c}$ & 206,2 c & $474 \mathrm{e}$ & $90 \mathrm{e}$ & $575 \mathrm{e}$ \\
\hline $\mathrm{S} 10$ & $13,7 \mathrm{~d}$ & $2,1 \mathrm{f}$ & $0,69 \mathrm{e}$ & $1,40 \mathrm{f}$ & $0,73 \mathrm{~d}$ & $0,14 \mathrm{~d}$ & $53,93 \mathrm{c}$ & $1595 \mathrm{~d}$ & $490 \mathrm{e}$ & $28 \mathrm{f}$ & $299 \mathrm{f}$ \\
\hline $\mathrm{CV}(\%)$ & 4,48 & 10,31 & 9,26 & 8,59 & 6,22 & 8,36 & 8,17 & 8,01 & 7,35 & 8,29 & 4,56 \\
\hline
\end{tabular}

${ }^{1}$ Médias seguidas da mesma letra, na coluna, não diferem estatisticamente entre si pelo teste de Scott-Knott $(\mathrm{P}>0,05)$. 
Experimento I: ${ }^{2} \mathrm{BIO}$ (biossólido), CAC (casca de arroz carbonizada), FC (fibra de coco triturada).

As mudas produzidas no tratamento S10 (substrato comercial florestal) apresentaram as maiores teores de $\mathrm{N}, \mathrm{Ca}, \mathrm{Mg}$ e $\mathrm{Mn}$, que pode ser explicado pelo fato deste substrato apresentar maiores concentrações destes nutrientes que os outros tratamentos (Tabela 2). O mesmo pode ser observado para as concentrações de $\mathrm{K}$ na parte aérea, onde os tratamentos M1 (40\% TS + $35 \% \mathrm{~EB})$ e $\mathrm{M} 7(40 \% \mathrm{TS}+35 \% \mathrm{EC})$ apresentaram as maiores médias, explicado pela ocorrência das maiores concentrações de $\mathrm{K}$ nestes resíduos.

As maiores concentrações de $\mathrm{P}$ da parte aérea das mudas foram obtidas nos tratamentos M6 $(60 \% \mathrm{TS}+15 \% \mathrm{CF}), \mathrm{M} 7(40 \% \mathrm{TS}+35 \% \mathrm{EC}), \mathrm{M} 8$ $(50 \% \mathrm{TS}+25 \% \mathrm{EC})$ e M9 $(60 \% \mathrm{TS}+15 \% \mathrm{EC})$. Para os substratos as maiores concentrações de $\mathrm{P}$ foram nos tratamentos com as maiores frações dos estercos (M1, M4 e M7), indicando que o substrato não apresentou influência direta nas concentrações de $\mathrm{P}$ na parte aérea das mudas.

As concentrações de micronutrientes na parte aérea da mudas, exceto o $\mathrm{Mn}$, não foram influenciados diretamente pelo substrato, uma vez que não houve relação das concentrações totais e disponíveis dos substratos com as concentrações de nutrientes nas plantas.

Cunha et al. (2006), trabalhando com Acacia mangium e Acacia auriculiformis, em substrato com terra de subsolo, areia e esterco bovino (1:1:1, v:v:v) encontraram, 90 dias após a semeadura, quantidades de 7,29 $\mathrm{mg}_{\text {planta }}{ }^{-1}$ de N, 4,92 mg.planta ${ }^{-1}$ de P, 9,17 mg planta ${ }^{-1}$ de K,
7,04 mg planta $^{-1}$ de Ca e 3,10 mg planta $^{-1}$ de $\mathrm{Mg}$, nas mudas de $A$. mangium. Para $A$. auriculiformis, os autores encontraram quantidades de $11,66 \mathrm{mg}_{\text {planta }}{ }^{-1}$ de $\mathrm{N}, 5,13 \mathrm{mg}$ planta $^{-1}$ de P, 16,94 mg planta ${ }^{-1}$ de K, 7,72 mg planta $^{-1}$ de Ca e 3,42 mg planta ${ }^{-1}$ de $\mathrm{Mg}$. Para as duas espécies de acácia, a quantidade dos macronutrientes foram maiores aos observados nas mudas dos tratamentos do presente estudo.

Em contrapartida, Villela (2007), avaliando substratos orgânicos na produção de mudas de Acacia mearnsii, encontrou valores abaixo dos obtidos no presente estudo: 0,067 a $0,277 \mathrm{mg}$ planta $^{-1}$ de N, 0,082 a 0,420 mg planta ${ }^{-1}$ de P, 0,070 a $0,310 \mathrm{mg}$ planta $^{-1}$ de $\mathrm{K}, 0,105$ a 0,545 mg planta ${ }^{-1}$ de Ca e 0,017 a 0,175 mg planta $^{-1}$ de $\mathrm{Mg}$. A provável explicação é que a concentração de nutrientes no tecido vegetal depende de alguns fatores, como: exigências nutricionais da espécie, idade da muda, época de coleta do tecido, disponibilidade de nutrientes do substrato.

$\mathrm{Na}$ Tabela 7, pode-se observar que a correlação entre cada nutriente no substrato e a concentração deste na parte aérea das mudas foi significativa e positiva para $\mathrm{N}\left(\mathrm{R}=0,81^{* *}\right), \mathrm{K}$ $\left(\mathrm{R}=0,69^{* *}\right), \mathrm{S}\left(\mathrm{R}=0,57^{*}\right)$ e $\mathrm{Cu}\left(\mathrm{R}=0,45^{*}\right)$; não significativa para $P\left(R=0,33^{n s}\right), C a\left(R=-0,04^{n s}\right)$, $\operatorname{Mg}\left(R=0,33^{\mathrm{ns}}\right), \mathrm{Zn}\left(\mathrm{R}=0,29^{\mathrm{ns}}\right), \mathrm{Fe}\left(\mathrm{R}=0,18^{\mathrm{ns}}\right)$ e $\mathrm{Mn}\left(\mathrm{R}=0,12^{\mathrm{ns}}\right)$; e significativa, porém negativa para $\mathrm{B}\left(\mathrm{R}=-0,44^{*}\right)$.

Tabela 7. Correlação entre as características químicas dos substratos e as concentrações de nutrientes na parte aérea das mudas de Tectona grandis produzidas nos substratos formulados com esterco de animais (experimento II).

Table 7. Correlation between chemical characteristic of substrates and nutrient concentrations in shoots of seedling of Tectona grandis produced on substrates formulated with animal manures (experiment II).

\begin{tabular}{|c|c|c|c|c|c|c|c|c|c|c|c|}
\hline & $\mathrm{N}$ & $\mathrm{P}$ & $\mathrm{K}$ & $\mathrm{Ca}$ & $\mathrm{Mg}$ & $S$ & $\mathrm{Zn}$ & $\mathrm{Fe}$ & $\mathrm{Mn}$ & $\mathrm{Cu}$ & B \\
\hline $\mathrm{TN}$ & $0,81 * *$ & $0,36^{\mathrm{ns}}$ & $0,06^{\mathrm{ns}}$ & $0,32^{\mathrm{ns}}$ & $0,43^{*}$ & $0,09^{\mathrm{ns}}$ & $0,07^{\mathrm{ns}}$ & $-0,76 * *$ & $0,79 * *$ & $0,03^{\mathrm{ns}}$ & $0,81 * *$ \\
\hline $\mathrm{TP}$ & $-0,70 * *$ & $0,33^{\mathrm{ns}}$ & $0,23^{\text {ns }}$ & $0,19^{\mathrm{ns}}$ & $-0,87 * *$ & $0,49 *$ & $0,56^{*}$ & $0,88 * *$ & $-0,79 * *$ & $0,51 *$ & $-0,43 *$ \\
\hline TK & $-0,20^{\mathrm{ns}}$ & $-0,05^{\mathrm{ns}}$ & $0,69 * *$ & $0,36^{\mathrm{ns}}$ & $-0,05^{\mathrm{ns}}$ & $0,75^{* *}$ & $-0,16^{\mathrm{ns}}$ & $0,18^{\mathrm{ns}}$ & $-0,06^{\mathrm{ns}}$ & $-0,31^{\mathrm{ns}}$ & $0,13^{\mathrm{ns}}$ \\
\hline Tca & $0,13^{\mathrm{ns}}$ & $0,15^{\mathrm{ns}}$ & $-0,90 * *$ & $-0,04^{\mathrm{ns}}$ & $-0,21^{\mathrm{ns}}$ & $-0,41^{*}$ & $0,18^{\mathrm{ns}}$ & $0,02^{\text {ns }}$ & $-0,33^{\mathrm{ns}}$ & $0,33^{\mathrm{ns}}$ & $0,10^{\mathrm{ns}}$ \\
\hline $\mathrm{TMg}$ & $0,81 * *$ & $0,44^{*}$ & $-0,59 *$ & $-0,01^{\mathrm{ns}}$ & $0,33^{\mathrm{ns}}$ & $-0,49 *$ & $0,29^{\mathrm{ns}}$ & $-0,72 * *$ & $0,64 * *$ & $0,37^{\mathrm{ns}}$ & $0,60 * *$ \\
\hline $\mathrm{TS}$ & $-0,40 *$ & $-0,06^{\mathrm{ns}}$ & $0,50 *$ & $0,61 * *$ & $-0,13^{\mathrm{ns}}$ & $0,57 *$ & $-0,11^{\mathrm{ns}}$ & $0,39^{\text {ns }}$ & $-0,33^{\mathrm{ns}}$ & $-0,32^{\mathrm{ns}}$ & $-0,12^{\mathrm{ns}}$ \\
\hline $\mathrm{TZn}$ & $0,12^{\mathrm{ns}}$ & $0,38^{\mathrm{ns}}$ & $-0,44^{\mathrm{ns}}$ & $0,11^{\mathrm{ns}}$ & $-0,01^{\mathrm{ns}}$ & $0,01^{\mathrm{ns}}$ & $0,29^{\mathrm{ns}}$ & $-0,04^{\mathrm{ns}}$ & $-0,28^{\mathrm{ns}}$ & $0,31^{\mathrm{ns}}$ & $0,39^{\text {ns }}$ \\
\hline $\mathrm{TFe}$ & $-0,10^{\mathrm{ns}}$ & $-0,31^{\mathrm{ns}}$ & $-0,15^{\mathrm{ns}}$ & $-0,16^{\mathrm{ns}}$ & $-0,28^{\mathrm{ns}}$ & $0,51 *$ & $-0,31^{\mathrm{ns}}$ & $0,18^{\mathrm{ns}}$ & $-0,39^{\mathrm{ns}}$ & $-0,24^{\mathrm{ns}}$ & $0,19^{\text {ns }}$ \\
\hline TMn & $0,33^{\mathrm{ns}}$ & $-0,45^{*}$ & $-0,77 * *$ & $-0,39^{\mathrm{ns}}$ & $0,20^{\mathrm{ns}}$ & $-0,49 *$ & $-0,45^{*}$ & $-0,33^{\mathrm{ns}}$ & $0,12^{\text {ns }}$ & $-0,26^{\mathrm{ns}}$ & $0,07^{\mathrm{ns}}$ \\
\hline $\mathrm{TCu}$ & $-0,82 * *$ & $0,36^{\mathrm{ns}}$ & $0,49 *$ & $0,29^{\text {ns }}$ & $-0,64 * *$ & $0,37^{\mathrm{ns}}$ & $0,60 * *$ & $0,87 * *$ & $-0,69 * *$ & $0,45^{*}$ & $-0,61 * *$ \\
\hline $\mathrm{TB}$ & $-0,77 * *$ & $0,01^{\mathrm{ns}}$ & $0,51 *$ & $0,42 *$ & $-0,57 *$ & $0,65^{* *}$ & $0,14^{\mathrm{ns}}$ & $0,82 * *$ & $-0,72 * *$ & $-0,01^{\mathrm{ns}}$ & $-0,44^{*}$ \\
\hline
\end{tabular}

Teste F: ${ }^{* *}$ significativo ao nível de $1 \%$ de probabilidade; ${ }^{*}$ significativo ao nível de $5 \%$ de probabilidade; ${ }^{\text {ns }}$ não significativo 
$(\mathrm{p}>0,05)$

A correlação entre as concentrações totais nos substratos formulados com estercos de animais e as concentrações totais na parte aérea das mudas, pode ser um indicativo de que não houve lixiviação dos nutrientes no substrato, uma vez que apenas para o B a correlação foi significativa e negativa. Apesar de altas concentrações totais de $\mathrm{P}$ disponibilizados pelos estercos de animais, a correlação foi não significativa. É possível que devido às altas proporções de terra de subsolo nestes tratamentos $(40,50$ e $60 \%)$, o P ficou adsorvido pelos argilominerais, indicando uma dificuldade de absorção deste nutriente pelas plantas (NOVAIS et al., 2007).

Analisando separadamente a quantidade de cada nutriente na parte aérea com a concentração total deste mesmo no nutriente no substrato, conforme a Tabela 8, a correlação foi significativa e positiva para $\mathrm{P}\left(\mathrm{R}=0,49^{*}\right), \mathrm{K}(\mathrm{R}=$ $\left.0,58^{*}\right), \mathrm{Zn}\left(\mathrm{R}=0,65^{* *}\right)$ e $\mathrm{Cu}\left(\mathrm{R}=0,56^{*}\right)$; não significativa para $\mathrm{N}\left(\mathrm{R}=-0,05^{\mathrm{ns}}\right), \mathrm{Ca}(\mathrm{R}=-$ $\left.0,06^{\mathrm{ns}}\right), \mathrm{Mg}\left(\mathrm{R}=0,00^{\mathrm{ns}}\right), \mathrm{S}\left(\mathrm{R}=-0,07^{\mathrm{ns}}\right), \mathrm{Fe}(\mathrm{R}=$ $\left.0,17^{\mathrm{ns}}\right)$ e $\mathrm{Mn}\left(\mathrm{R}=0,09^{\mathrm{ns}}\right)$; e significativa, porém negativa para $\mathrm{B}(\mathrm{R}=-0,53 *)$.

Tabela 8. Correlação entre as características químicas dos substratos e as quantidades de nutrientes na parte aérea das mudas de Tectona grandis produzidas nos substratos formulados com estercos de animais (experimento II).

Table 8. Correlation between the chemical characteristics of the substrates and the amounts of nutrients in shoots of seedlings of Tectona grandis produced on substrates formulated with animal manures (experiment II).

\begin{tabular}{|c|c|c|c|c|c|c|c|c|c|c|c|}
\hline & $\bar{N}$ & $\mathrm{P}$ & $\bar{K}$ & $\mathrm{Ca}$ & $\mathrm{Mg}$ & $\mathrm{S}$ & $\mathrm{Zn}$ & $\mathrm{Fe}$ & $\mathrm{Mn}$ & $\mathrm{Cu}$ & B \\
\hline$\overline{\mathrm{AN}}$ & $-0,05^{\mathrm{ns}}$ & $0,44^{*}$ & $0,16^{\mathrm{ns}}$ & $-0,07^{\mathrm{ns}}$ & $-0,01^{\mathrm{ns}}$ & $-0,20^{\mathrm{ns}}$ & $0,59^{*}$ & $0,02^{\mathrm{ns}}$ & $0,21^{\mathrm{ns}}$ & $0,48^{*}$ & $-0,21^{\mathrm{ns}}$ \\
\hline AP & $-0,34^{\mathrm{ns}}$ & $0,49 *$ & $0,16^{\mathrm{ns}}$ & $0,00^{\mathrm{ns}}$ & $-0,33^{\mathrm{ns}}$ & $-0,04^{\mathrm{ns}}$ & $0,72 * *$ & $0,37^{\mathrm{ns}}$ & $-0,16^{\mathrm{ns}}$ & $0,62 * *$ & $39^{\text {ns }}$ \\
\hline AK & $-0,34^{\mathrm{ns}}$ & $0,24^{\mathrm{ns}}$ & $0,58 *$ & $-0,01^{\mathrm{ns}}$ & $-0,05^{\mathrm{ns}}$ & $0,12^{\mathrm{ns}}$ & $0,43 *$ & $0,24^{\mathrm{ns}}$ & $0,11^{\mathrm{ns}}$ & $0,23^{\mathrm{ns}}$ & $-0,39^{\mathrm{ns}}$ \\
\hline $\mathrm{ACa}$ & $-0,09^{\mathrm{ns}}$ & $0,45^{*}$ & $-0,14^{\mathrm{ns}}$ & $-0,06^{\mathrm{ns}}$ & $-0,11^{\mathrm{ns}}$ & $-0,34^{\mathrm{ns}}$ & $0,63 * *$ & $0,11^{\mathrm{ns}}$ & $0,00^{\mathrm{ns}}$ & $0,57 *$ & $-0,25^{\mathrm{ns}}$ \\
\hline $\mathrm{AMg}$ & $13^{\mathrm{ns}}$ & $0,54 *$ & $-0,17^{\mathrm{ns}}$ & $-0,08^{\mathrm{ns}}$ & $0,00^{\mathrm{ns}}$ & $-0,36^{\mathrm{ns}}$ & $0,65^{* *}$ & $-0,10^{\mathrm{ns}}$ & $0,21^{\mathrm{ns}}$ & $0,62 * *$ & $-0,04^{\mathrm{ns}}$ \\
\hline AS & $-0,33^{\mathrm{ns}}$ & $0,28^{\text {ns }}$ & $0,35^{\mathrm{ns}}$ & $0,07^{\mathrm{ns}}$ & $-0,08^{\mathrm{ns}}$ & $-0,07^{\mathrm{ns}}$ & $0,49^{*}$ & $0,27^{\mathrm{ns}}$ & $0,02^{\mathrm{ns}}$ & $0,30^{\mathrm{ns}}$ & $-0,46^{*}$ \\
\hline $\mathrm{AZn}$ & $-0,09^{\mathrm{ns}}$ & $0,49 *$ & $0,01^{\mathrm{ns}}$ & $-0,10^{\mathrm{ns}}$ & $-0,05^{\mathrm{ns}}$ & $-0,24^{\mathrm{ns}}$ & $0,65 * *$ & $0,08^{\mathrm{ns}}$ & $0,05^{\mathrm{ns}}$ & $0,57 *$ & $-0,19^{\mathrm{ns}}$ \\
\hline $\mathrm{AFe}$ & $-0,21^{\mathrm{ns}}$ & $0,34^{\mathrm{ns}}$ & $0,17^{\mathrm{ns}}$ & $-0,13^{\mathrm{ns}}$ & $-0,10^{\mathrm{ns}}$ & $-0,14^{\mathrm{ns}}$ & $0,55^{*}$ & $0,17^{\mathrm{ns}}$ & $0,05^{\mathrm{ns}}$ & $0,44^{*}$ & $-0,34^{\mathrm{ns}}$ \\
\hline AMn & $-0,08^{\mathrm{ns}}$ & $0,28^{\mathrm{ns}}$ & $-0,10^{\mathrm{ns}}$ & $-0,14^{\mathrm{ns}}$ & $-0,03^{\mathrm{ns}}$ & $-0,37^{\mathrm{ns}}$ & $0,47 *$ & $0,06^{\mathrm{ns}}$ & $0,09^{\mathrm{ns}}$ & $0,42 *$ & $-0,32^{\mathrm{ns}}$ \\
\hline $\mathrm{ACu}$ & $-0,30^{\mathrm{ns}}$ & $0,46^{*}$ & $0,22^{\mathrm{ns}}$ & $-0,02^{\mathrm{ns}}$ & $-0,21^{\mathrm{ns}}$ & $-0,10^{\mathrm{ns}}$ & $0,67 * *$ & $0,30^{\mathrm{ns}}$ & $-0,07^{\mathrm{ns}}$ & $0,56^{*}$ & $-0,39^{\mathrm{ns}}$ \\
\hline $\mathrm{AB}$ & $-0,45^{*}$ & $0,30^{\mathrm{ns}}$ & $0,38^{\mathrm{ns}}$ & $0,07^{\mathrm{ns}}$ & $-0,21^{\mathrm{ns}}$ & $0,02^{\mathrm{ns}}$ & $0,54^{*}$ & $0,41 *$ & $-0,11^{\mathrm{ns}}$ & $0,36^{\mathrm{ns}}$ & $-0,53 *$ \\
\hline
\end{tabular}

Teste F: ** significativo ao nível de $1 \%$ de probabilidade; * significativo ao nível de $5 \%$ de probabilidade; ${ }^{\text {ns }}$ não significativo $(\mathrm{p}>0,05)$.

Como comentado anteriormente, é provável que ocorreu lixiviação de alguns nutrientes, e a do Boro se mostrou a mais evidente. A correlação indicou que, comparando as concentrações totais no substrato com as concentrações e quantidades deste nutriente na parte aérea, a correlação foi significativa e negativa.

\section{Conclusões}

As maiores concentrações e quantidades de nutrientes na parte aérea das mudas de $T$. grandis foram obtidos no tratamento com $80 \%$ de biossólido associado a $20 \%$ de casca de arroz carbonizada, no experimento com biossólido.

No estudo com estercos de animais, a maior quantidade de nutrientes foi observada no tratamento com $35 \%$ de cama de frango. As maiores concentrações de $\mathrm{K}, \mathrm{S}$ e $\mathrm{Zn}$ foram encontrados nos estercos de codorna e esterco bovino, enquanto as de $\mathrm{P}, \mathrm{Cu}$ e $\mathrm{B}$ no esterco de codorna.

A utilização de substratos alternativos pode ser indicada para produção de mudas florestais, contribuindo nas características físicas e químicas dos substratos formados e consequentemente na nutrição das plantas.

A correlação entre cada nutriente no substrato 
e a concentração do mesmo na parte aérea das mudas foi significativa e positiva para $\mathrm{N}(\mathrm{R}=$ $\left.0,81^{* *}\right), \mathrm{K}\left(\mathrm{R}=0,69^{* *}\right), \mathrm{S}\left(\mathrm{R}=0,57^{*}\right)$ e $\mathrm{Cu}(\mathrm{R}=$ $\left.0,45^{*}\right)$. Para o acúmulo de cada nutriente na parte aérea com a concentração total deste mesmo no substrato, a correlação foi significativa e positiva para $\mathrm{P}\left(\mathrm{R}=0,49^{*}\right), \mathrm{K}(\mathrm{R}=$ $\left.0,58^{*}\right), \mathrm{Zn}\left(\mathrm{R}=0,65^{* *}\right)$ e $\mathrm{Cu}(\mathrm{R}=0,56)$

\section{Referências}

ABRAF - ASSOCIAÇÃO BRASILEIRA DE PRODUTORES DE FLORESTAS PLANTADAS. Anuário Estatístico da ABRAF 2013: ano base 2012. Brasília: ABRAF, $142 \mathrm{p}$.

ALVAREZ V., V. H.; ROSCOE, R.; KURIHARA, C. H.; PEREIRA, N. de F. Enxofre. In: NOVAIS, R. F.; ALVAREZ V., V. H.; BARROS, N. F. de; FONTES, R. L. F.; CANTARUTTI, R. B.; NEVES, J. C. L. (Eds.). Fertilidade do solo. Viçosa: SBCS, 2007. p. 595-644.

ALVAREZ V., V.H.; NOVAIS, R. F.; BARROS, N. F.; CANTARUTTI, R. B.; LOPES, A. S. Interpretação dos resultados das análises de solos. In: RIBEIRO, A. C.; GUIMARÃES, P. T. G.; ALVAREZ V., V. H. (Ed.). Recomendação para o uso de corretivos e fertilizantes em Minas Gerais. $5^{\text {a }}$. Aproximação. Viçosa: Comissão de Fertilidade do Solo do Estado de Minas Gerais, 1999. p.2532.

AZEVEDO, R.A.; FAQUIN, V.; FERNANDES, L.R. Adsorção de boro em solos de várzea do Sul de Minas Gerais. Pesquisa Agropecuária Brasileira, v.36, p.957-964, 2001.

BRASIL. Ministério do Meio Ambiente. Conselho Nacional do Meio Ambiente. Resolução CONAMA no . 375. Define critérios e procedimentos, para o uso agrícola de lodos de esgoto gerados em estações de tratamento de esgoto sanitário e seus produtos derivados, e dá outras providências. Diário Oficial da República Federativa do Brasil, Brasília n. 167, p.141-146, 30 de ago 2006.

CRUZ, C. A. F.; PAIVA, H. N.; GOMES, K. C. O.; GUERRERO, C. R. A. Efeito de diferentes níveis de saturação por bases no desenvolvimento e qualidade de mudas de ipê roxo (Tabebuia impetiginosa (Mart.) Standley). Revista Scientia Forestalis, Piracicaba, n. 66, p. 100-107, 2004.

CUNHA, A.M.; CUNHA, G.M.; SAMENTO, R.A.; CUNHA, G.M.; AMARAL, J.F.T. Efeito de diferentes substratos sobre $\mathrm{O}$ desenvolvimento de mudas de Acacia sp. Revista Árvore, Viçosa, v.30, p.207-214, 2006.

D’AVILA, F.S. Efeito do fósforo, nitrogênio e potássio na produção de mudas clonais de eucalipto. 2008. 62 f. Dissertação (Mestrado em Ciência Florestal) - Universidade Federal de Viçosa, Viçosa.

EMPRESA BRASILEIRA DE PESQUISA AGROPECUÁRIA - EMBRAPA. Centro Nacional de Pesquisa de Solos. Sistema brasileiro de classificação de solos. 2.ed. Rio de Janeiro, 2006. 306p.

EMPRESA BRASILEIRA DE PESQUISA AGROPECUÁRIA - EMBRAPA. Manual de análises químicas de solos, plantas e fertilizantes. Brasília: Embrapa Informação Tecnológica, 2009. 627p.

ESPÍRITO SANTO. Secretaria de Estado de Ações Estratégicas e Planejamento. Informações municipais do Estado do Espírito Santo, 1994. Vitória: Departamento Estadual de Estatística, 1994. v. 1. 803 p.

FIGUEIREDO, E.O.; OLIVEIRA, L.C. de; BARBOZA, L.K.F. Teca (Tectona grandis L.f.): principais perguntas do futuro empreendedor florestal. Rio Branco: Embrapa Acre, 2005. 87 p. (Embrapa Acre. Documentos, 97). 
FONSECA, F. A.; LELES, P. S. S.; SAGGIN JÚNIOR, O. J.; SILVA, E. M. R.; OLIVEIRA NETO, S. N. Produção de mudas de Acacia mangium e Mimosa artemisiana utilizando resíduos urbanos como substratos, associados a fungos micorrízicos arbusculares. Boletim de Pesquisa e Desenvolvimento. Embrapa Agrobiologia, v. 67, 2010.

NOVAIS, R.F.; SMYTH, T.J.; NUNES, F.N. Fósforo. In: NOVAIS, R.F.; ALVAREZ V., V.H.; BARROS, N.F.; FONTES, R.L.F.; CANTARUTTI, R.B.; NEVES, J.C.L (Ed.). Fertilidade do Solo. Viçosa: Sociedade Brasileira de Ciência do Solo, 2007. p.471-550.

ROCHA, J. H. T.; BACKES, C.; DIOGO, F. A.; PASCOTTO, C. B.; BORELLI, K. Composto de lodo de esgoto como substrato para mudas de eucalipto. Pesquisa florestal brasileira. v. 33, n. 73, 2013.

ROSOLEM, C.A.; BÍSCARO, T. Adsorção e lixiviação de boro em Latossolo VermelhoAmarelo. Pesquisa Agropecuária Brasileira, v.42, n.10, p.1473-1478, 2007.

SILVA, W. G. da; TUCCI, C. A. F.; HARA, F. A. S.; SANTOS, R. A. C. dos. Efeito de micronutrientes sobre o crescimento de mudas de mogno (Swietenia Macrophilla King) em Latossolo amarelo. Acta Amazonica, Manaus, v. 37, p.371-376, 2007.

TRIGUEIRO, R.M.; GUERRINI, I.A. Uso de biossólido como substrato para produção de mudas de eucalipto. Revista Scientia Florestalis, Piracicaba, v.64, p.150-162, 2003.

VILLELA, A.T. Avaliação de substratos orgânicos na produção de mudas de acácia negra (Acacia mearnsii de Wild). 2007. 62 f. Dissertação (Mestrado em Agronomia) Universidade Federal de Pelotas, Pelotas. 